\title{
Applying Dynamic Change Impact Analysis in Component-based Architecture Design
}

\author{
Tie Feng \\ College of Computer Science and Technology \\ Jilin University, China \\ Changchun Jilin 130012 \\ fengtie@jlu.edu.cn
}

\begin{abstract}
Change impact analysis plays an important role in maintenance and evolution of component-based software architecture. Viewing component replacement as a change to composition-based software architecture, this paper proposes a component interaction trace based approach to support dynamic change impact analysis at software architecture level. Given an architectural change, our approach determines the architecture elements causing the change and impacted by the change. Firstly, component-based software architecture and component interaction trace are defined. An algorithm for generating component interaction trace from static structure model of software architecture and UML sequence diagram is provided. Secondly, the taxonomy of changes on composition-based software architecture is presented, according to which a set of impact rules are suggested to determine the transfer of the changes in component and among components. Thirdly, by performing slicing on component interaction traces according to impact rules, the impact analysis results are obtained. Finally, the architecture design of SOCIAT, a tool supporting our approach, is developed and explained.
\end{abstract}

Keywords: Change impact analysis, software architecture, component composition, program slicing

\section{Introduction}

Frequent changes and expansion to legacy system cause unexpected influence on the other parts of it. Component-based software engineering is an effective way to alleviate this problem to some extent, because the internal information of the component is not available to the public and components only communicate through their interfaces.

However, it is never a simple procedure to retrieve proper components from component repository and replace the old ones with them so that changed system functionality and performance are obtained. In many cases, the existing components have less or more services

\author{
Jonathan I. Maletic \\ Department of Computer Science \\ Kent State University, US \\ Kent Ohio 44242 \\ \{hkagdi, collard, jmaletic\}@cs.kent.edu
}

provided and requiring than expected ones. Besides customizing the retrieved components or developing new components, change impact analysis is also an effective way to support maintenance and evolution of component based software development by determining subsequent changes, if component replacement is considered as a change to legacy system.

Change impact analysis is to identify the potential consequences of a change or estimate what needs to be modified to accomplish a change [1]. Much effort has been made to study static and dynamic change impact analysis technologies at the code level of software systems. However, very few techniques have been proposed to support change impact analysis on component composition at software architecture level, and much fewer research have been conducted to support dynamic change impact analysis on component composition at this abstract level.

On the other hand, composition based software architecture of a system defines its high-level structure, exposing its gross organization as a collection of interacting components [2]. It offers a way to partition complex systems into well-defined parts $[3,4]$. We try to apply change impact analysis to composition based software architecture in order to support software architecture maintenance and evolution.

It is important to understand dynamic analysis on component composition at architecture level to determine our research scope. Our approach considers the system dynamic behaviors at a rather higher level and focus on studying effects on software product of adding or removing components at runtime. Starting from component interaction traces describing the interaction sequences among components through their interfaces, our approach study the impacts of changes on architectural elements according to a special system execution with a set of given inputs or interaction triggered by user.

In order to develop dynamic change impact analysis technique on composition based software architecture, interaction mechanism and composition model among components of architecture are required. In this paper, 
components are integrated together through interfaces and methods in them. Moreover, a dynamic component composition model is defined and derived from static structure model of component composition and a set of UML sequence diagrams describing object interaction. Then the dynamic change impact analysis is accomplished through slicing on component interaction traces. The slicing criterion is constructed on intracomponent and inter-component impact rules for taxonomy of changes of interface and methods of components.

\section{Composition based software architecture}

The software architecture of a system is the structure or structures of the system, which comprise software components, the externally visible properties of those components, and the relationships among them. In this paper, composition based software architecture is defined as a special kind of software architecture in which component is considered as the first-class entity and connectors is not obviously modeled. Components are integrated together through interfaces to achieve special system functionality.

\subsection{Component and interface}

Each component comprises a set of interfaces and the application logic [5]. Interfaces can be divided into two categories: provider interface providing services to other components or external environment, and requirement interface requiring services from other components or external environment. Each interface is consist of a set of methods indicating the identifications of provided services or required ones. The structure of an example component $\mathrm{C}$ is shown in Fig. 1. Component $\mathrm{C}$ has 2 provider interfaces $\left(\mathrm{I}_{\mathrm{p} 1}\right.$ and $\left.\mathrm{I}_{\mathrm{p} 2}\right)$ and 2 requirement interfaces $\left(\mathrm{I}_{\mathrm{r} 1}\right.$ and $\left.\mathrm{I}_{\mathrm{r} 2}\right)$. Interface $\mathrm{I}_{\mathrm{p} 1}$ is composed with 2 methods, each of which indicates a service $\mathrm{C}$ provides through interface $\mathrm{I}_{\mathrm{p} 1}$.

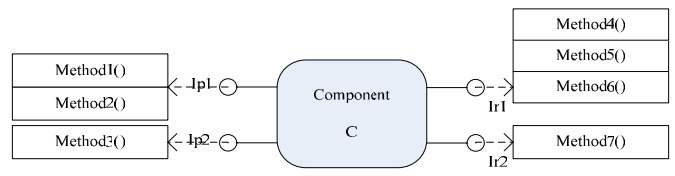

Fig. 1. An example of component

To demonstrate our approach, we introduce following primitives in Table 1.
Table 1. Primitive for Component, Interface and Method

\begin{tabular}{|l|l|l|}
\hline Primitives & Parameters & Return \\
\hline Interface & C: Component & A set of interfaces of component C \\
\hline PorR & I: Interface & $\begin{array}{l}\text { Provider if I is a provider interface, } \\
\text { Requirement otherwise }\end{array}$ \\
\hline Method & I: Interface & A set of methods of interface I \\
\hline
\end{tabular}

Definition 1: Initial Component. Let $\mathrm{C}$ be a component, if for $\forall \mathrm{I} \in \operatorname{Interface}(\mathrm{C})$, PorR(I)=Requirement, then we say $\mathrm{C}$ is an initial component.

Definition 2: Final Component. Let $\mathrm{C}$ be a component, if for $\forall \mathrm{I} \in \operatorname{Interface}(\mathrm{C})$, PorR(I)=Provider, then we say $\mathrm{C}$ is an final component.

Components communicate through their interfaces and there are three kinds of relationships between two interfaces: interface equivalence, interface satisfaction and interface inclusion.

Definition 3: Interface Equivalence. Let $\mathrm{C}_{1}$ and $\mathrm{C}_{2}$ be two components, if $\exists \mathrm{I}_{1} \in \operatorname{Interface}\left(\mathrm{C}_{1}\right) \wedge \mathrm{I}_{2} \in$ Interface $\left(\mathrm{C}_{2}\right)$, so that $\left(\operatorname{PorR}\left(\mathrm{I}_{1}\right)=\operatorname{PorR}\left(\mathrm{I}_{2}\right)\right) \wedge\left(\operatorname{Method}\left(\mathrm{I}_{1}\right)=\right.$ $\left.\operatorname{Method}\left(\mathrm{I}_{2}\right)\right)$, then we say interface $\mathrm{I}_{1}$ is equivalent to interface $\mathrm{I}_{2}$, which is denoted as $\mathrm{I}_{1} \equiv \mathrm{I}_{2}$.

Definition 4: Interface Satisfaction. Let $C_{1}$ and $C_{2}$ be two components, if $\exists \mathrm{I}_{1} \in \operatorname{Interface}\left(\mathrm{C}_{1}\right) \wedge \mathrm{I}_{2} \in \operatorname{Interface}\left(\mathrm{C}_{2}\right)$, so that $\left(\operatorname{PorR}\left(\mathrm{I}_{1}\right)=\right.$ Provider $\wedge \operatorname{PorR}\left(\mathrm{I}_{2}\right)=$ Requirement $) \wedge$ $\left(\operatorname{Method}\left(\mathrm{I}_{1}\right) \supseteq \operatorname{Method}\left(\mathrm{I}_{2}\right)\right)$, then we say interface $\mathrm{I}_{1}$ satisfies interface $I_{2}$, which is denoted as $I_{1} \triangleright I_{2}$.

Definition 5: Interface Inclusion. Let $C_{1}$ and $C_{2}$ be two components, if $\exists \mathrm{I}_{1} \in \operatorname{Interface}\left(\mathrm{C}_{1}\right) \wedge \mathrm{I}_{2} \in \operatorname{Interface}\left(\mathrm{C}_{2}\right)$, so that $\left(\operatorname{PorR}\left(\mathrm{I}_{1}\right)=\operatorname{PorR}\left(\mathrm{I}_{2}\right)\right) \wedge\left(\operatorname{Method}\left(\mathrm{I}_{1}\right) \supseteq \operatorname{Method}\left(\mathrm{I}_{2}\right)\right)$, then we say interface $I_{1}$ includes interface $I_{2}$, which is denoted as $\mathrm{I}_{1} \geq \mathrm{I}_{2}$.

From above definitions, it is obvious that interface equivalence is a special example of interface inclusion. That is to say if $I_{1} \geq I_{2} \wedge I_{2} \geq I_{1}$, then $I_{1} \equiv I_{2}$.

\subsection{Static structure model of composition}

Static structure model is composed of components and connection among components according to interface inclusion and interface satisfaction. We develop two composition rules to configure components as follows.

Rule 1: Let $I_{1}$ and $I_{2}$ are two interfaces of two different components $\mathrm{C}_{1}$ and $\mathrm{C}_{2}$, if it is satisfied that $\left(\mathrm{I}_{1} \geq \mathrm{I}_{2}\right)$ $\wedge\left(\operatorname{PorR}\left(\mathrm{I}_{1}\right)=\operatorname{PorR}\left(\mathrm{I}_{2}\right)=\right.$ Provider $)$, then we create a new provider interface $I^{\prime}=I_{1}$.

Rule 2: Let $I_{1}$ and $I_{2}$ are two interfaces of two different components $\mathrm{C}_{1}$ and $\mathrm{C}_{2}$, if it is satisfied that $\mathrm{I}_{1} \triangleright \mathrm{I}_{2}$, then the binding of the two interfaces indicates that the service required by $\mathrm{C}_{2}$ through interface $\mathrm{I}_{2}$ is provided by $\mathrm{C}_{1}$ through interface $\mathrm{I}_{1}$.

For example, Fig. 2 shows an example of application of rule 1 and rule 2 . Rule 1 is applied between $\mathrm{I}_{\mathrm{p} 1}$ and $\mathrm{I}_{\mathrm{p} 2}$ and a new provider interface $I_{p}$ is created. Rule 2 is applied between $\mathrm{I}_{\mathrm{p}}$ and $\mathrm{I}_{\mathrm{r} 3}, \mathrm{I}_{\mathrm{p} 3}$ and $\mathrm{I}_{\mathrm{r} 1}, \mathrm{I}_{\mathrm{p} 4}$ and $\mathrm{I}_{\mathrm{r} 2}$. Of course, 
the application of rules must meet the prerequisite of the rules.

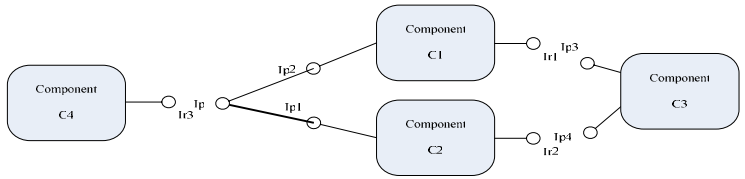

Fig. 2. An example of static structure model of compositionbased software architecture

\subsection{Dynamic interaction model of composition}

Dynamic interaction model represents the interactions among components through their interfaces. Taking the static structure model shown in Fig. 2 for instance, two possible dynamic interactions among components are shown in Fig. 3 (a) and Fig. 3 (b). In this paper, we introduce a new concept of component interaction trace to describe the dynamic behavior model of composition. It is the foundation of our approach on change impact analysis.



Fig. 3 (a). One possibility

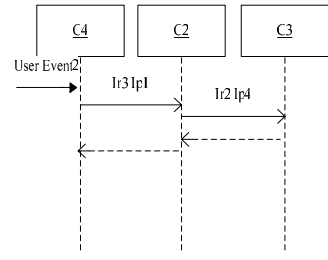

(b). Another possibility
Definition 6: Component Interaction Trace (CIT). $<$ CIT $>=<$ CompName $>$ $\mid<$ CIT $>$ " (" $<$ InterfacePair $>$ ")" $<$ CompName $>$ $\mid<$ CIT $><$ Mark $>$ "(" $<$ InterfacePair $>$ ")" $<$ CompName $>$ $<$ Mark $>=$ "r"|"“r" $<$ Mark $>$

$<$ InterfacePair $>=<$ RequirementIName $>$ ":" $<$ ProvIName $>$

" $r$ " indicates the return of control.

For example, the component interaction trace for dynamic interaction models shown in Fig. 3 (a) is $\mathrm{C}_{4}$ $\left(\mathrm{I}_{\mathrm{r} 3}: \mathrm{I}_{\mathrm{p} 2}\right) \mathrm{C}_{1}\left(\mathrm{I}_{\mathrm{r} 1}: \mathrm{I}_{\mathrm{p} 3}\right) \mathrm{C}_{3}$ rr $\left(\mathrm{I}_{\mathrm{r} 3}: \mathrm{I}_{\mathrm{p} 1}\right) \mathrm{C}_{2}\left(\mathrm{I}_{\mathrm{r} 2}: \mathrm{I}_{\mathrm{p} 4}\right) \mathrm{C}_{3}$ rr and the component interaction for Fig.3 (b) is $\mathrm{C}_{4}\left(\mathrm{I}_{\mathrm{r} 3}: \mathrm{I}_{\mathrm{p} 1}\right) \mathrm{C}_{2}\left(\mathrm{I}_{\mathrm{r} 2}: \mathrm{I}_{\mathrm{p} 4}\right)$ $\mathrm{C}_{3} \mathrm{rr}$.

In practical development of software systems using UML, the static structure model of composition and the interaction model of objects could be modeled through modified UML component diagrams and sequence diagrams. However, there is no automatic method generating dynamic interaction model of composition and component interaction trace. To perform dynamic change impact analysis on CIT, we present an algorithm as follow to automatically generate one CIT from static structure model and sequence diagram.
Input: Static Structure Model(SSM) and a Sequence

Diagram

Output: CIT describing component dynamic interactions

1 Traverse the sequence diagram to create a vector $V$ in which each element is a message.

2 Every message has the structure of $<$ BeginObject, MessageName, EndObject $>$.

3 CIT=Component which includes the BeginObject of the first message.

4 For each $V[i]$

5 For each component $C_{j}$ in $S S M$

$6 \quad$ For each service interface $\mathrm{Ip}_{k}$ of $C_{j}$

7 If V[i].MessageName $\in$ Method $\left(\operatorname{Ip}_{k}\right)$

$8 \quad$ For each component $C_{m}$ in SSM satisfying that $\exists$ Ir $\in$ Interface $\left(C_{m}\right) \wedge\left(I p_{k} \triangleright I r\right)$

$9 \quad$ Put all methods in all service interfaces of $C_{m}$ into a set $S_{l}$.

$10 u=i$; Put all messageNames received by $V[u]$.BeginObject currently into a set $S_{2}$.

11 While $(u>=0) \wedge\left(S_{1} \cap S_{2}=\Phi\right)$

$12 \quad u=u-1$.

13 If (V[u].BeginObject $\left.\notin C_{m}\right)$ $C I T=C I T+" r "$.

$14 \quad$ Put all messageNames received by $V[u]$.BeginObject into $S_{2}$

15

If $(u>=0)$

$$
C I T=C I T+"\left("+I r+":,+I p_{k}+"\right),+C_{j .} .
$$

17 Return CIT

In this algorithm, it is very easy to determine current component and its current provider interface by continuously analyzing the object message trace (line 1 to line 7). However, to determine which requirement interface of previous component is invoking service from current provider interface of current component, we have to retrace the sequence diagram until the message indirectly sent to current component and indirectly triggering the provider interface of current component is found (line 8 to line 15).

This algorithm is provider interface driven because the requirement interface information is not described in sequence diagram although it is defined in static structure model of composition.

\section{Changes and impacts on composition based software architecture}

First of all, we give taxonomy of changes on composition. Then the transition rules for intercomponent and intra-component are studied according to various changes.

\subsection{Taxonomy of changes}


Our approach focuses on the changes and impacts of elements of components. Concretely, the provider interfaces, requirement interfaces, methods in provider interfaces and methods in requirement interfaces of components are researched.

- Atomic Changes. An atomic change is that applied on components or interfaces and can't be decomposed into smaller changes. The atomic changes on composition based software architecture are listed in table2, which are self-explanatory.

Table 2. Atomic Changes on Composition Based Software Architecture

\begin{tabular}{|l|l|}
\hline AEPI & Add an Empty Provider Interface to component \\
\hline DEPI & Delete an Empty Provider Interface from component \\
\hline AERI & Add an Empty Requirement Interface to component \\
\hline DERI & Delete an Empty Requirement Interface from component \\
\hline AMPI & Add a Method signature to a Provider Interface \\
\hline DMPI & Delete a Method signature from a Provider Interface \\
\hline AMRI & Add a Method signature to a Requirement Interface \\
\hline DMRI & Delete a Method signature from a Requirement Interface \\
\hline
\end{tabular}

- Composite Change. A composite change, obtained through iterative transitions of atomic changes, is much more practical in change impact analysis. Sometimes, an individual atomic change to software architecture has not practical meanings. For example, typical addition of an empty provider interface is often followed by some additions of methods to it so that new services could be provided through new interface. Four kinds of practical composite changes on composition base software architecture are studied and listed in Table 3.

Table 3. Typical Composite Changes on Composition Based Software Architecture

\begin{tabular}{|l|l|}
\hline API $=\mathrm{AEPI}+\mathrm{n} * \mathrm{AMPI}$ & $\begin{array}{l}\text { Add an empty Provide Interface to component, } \\
\text { then add } \mathrm{n} \text { methods to it }\end{array}$ \\
\hline $\mathrm{DPI}=\mathrm{n} * \mathrm{DMPI}+\mathrm{DEPI}$ & $\begin{array}{l}\text { Delete } \mathrm{n} \text { methods from an provider interface, } \\
\text { then delete that interface }\end{array}$ \\
\hline $\mathrm{ARI}=\mathrm{AERI}+\mathrm{n} * \mathrm{AMRI}$ & $\begin{array}{l}\text { Add an empty Requirement Interface to } \\
\text { component, then add } \mathrm{n} \text { methods to it }\end{array}$ \\
\hline $\mathrm{DRI}=\mathrm{n} * \mathrm{DMRI}+\mathrm{DERI}$ & $\begin{array}{l}\text { Delete } \mathrm{n} \text { methods from an Requirement interface, } \\
\text { then delete that interface }\end{array}$ \\
\hline
\end{tabular}

Therefore, our approach focuses on 8 kinds of practical changes to software architecture elements: API, DPI, ARI, DRI, AMPI, DMPI, AMRI and DMRI. The first 4 changes are related to interfaces of components and the later 4 are related to methods of interfaces.

\subsection{Intra-component impact rule and inter- component impact rule}

As mentioned in above section, our approach focuses on the changes such as adding and deleting interfaces and methods. It is time to answer following questions: (1) what impacts would be produced on the same component and neighbor components? (2) How does the impact transmit?

- Intra-Component impact transition rule. Generally, to provide a service, a component would always require some services provided by other components. At the same time, a required service is always used to implement one or more provided services. Except for initial components and final components (see definition 1 and 2), a many-tomany mapping exists between methods in requirement interface and methods in provider interface. To initial components or final components, the changes has no impact on the other interfaces and methods of the same component because they have only requirement interfaces or provider interfaces.

For example, table 4 shows a possible intra-component method mapping of a single component shown in Fig. 1. C depends on Method5(), Method6() and Method7() to provide Method1().C depends on Method4()to provide Method2(). According to table 4, it is easy to conclude that interface Ip1 depends on Ir1, Ir2, while Interface Ip2 depends on Ir1 shown in Fig. 4.

Table 4. A Mapping among Methods between Provider Interfaces and Requirement Interfaces

\begin{tabular}{|c|c|c|c|c|}
\hline & Method4() & Method5() & Method6() & $\begin{array}{c}\text { Method7( } \\
)\end{array}$ \\
\hline Method1() & & $\mathrm{X}$ & $\mathrm{X}$ & $\mathrm{X}$ \\
\hline Method2() & $\mathrm{X}$ & & & $\mathrm{X}$ \\
\hline Method3() & & $\mathrm{X}$ & & \\
\hline
\end{tabular}

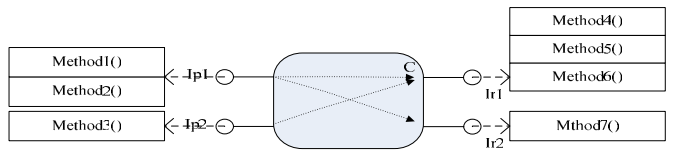

Fig. 4. Dependency among interfaces in a single component

With information on method mapping and interface dependency, it is easy to determine the intra-component impacts. For example, the Ip1 will be impacted by deleting the interface Ir2. Both interface Ip1 and Ip2 will be impacted by deleting Ir1. Generally,

Rule 3: DMRI or DRI cause DMPI or DPI on the same component.

Rule 4: AMPI or API cause AMRI or ARI on the same component.

- Inter-Component impact transition rule.

Definition 7: Neighbor. Let $\mathrm{C}_{1}$ and $\mathrm{C}_{2}$ be two components, if $\exists \mathrm{I}_{1} \in$ Interface $\left(\mathrm{C}_{1}\right) \wedge \mathrm{I}_{2} \in \operatorname{Interface}\left(\mathrm{C}_{2}\right) \wedge$ $\mathrm{I}_{2} \triangleright \mathrm{I}_{1}$, then we say $\mathrm{C}_{1}$ is left Neighbor of $\mathrm{C}_{2}$ and $\mathrm{C}_{2}$ is right neighbor of $\mathrm{C}_{1}$.

According to definition 7 , the concept of neighbor is for static structure model of component composition. For any segment in component interaction trace with form 
$\mathrm{C}_{\mathrm{m}}\left(\mathrm{I}_{\mathrm{ri}}: \mathrm{I}_{\mathrm{pj}}\right) \mathrm{C}_{\mathrm{n}}\left(\mathrm{I}_{\mathrm{rs}}: \mathrm{I}_{\mathrm{pt}}\right) \mathrm{C}_{\mathrm{o}}$, there exist that $\mathrm{C}_{\mathrm{m}}$ is the left neighbor of $C_{n}$ and $C_{o}$ is the right neighbor of $C_{n}$. It is obvious that the requirement interface $\mathrm{I}_{\mathrm{ri}}$ of component $\mathrm{C}_{\mathrm{m}}$ will be impacted by deleting the Ipj or methods in and $I_{p j}$. Provider interface $I_{p t}$ of component $C_{o}$ will be impact by adding methods into requirement interface Irs of component $C_{n}$. For the trace segment with return marks, it is necessary to refer to static structure model to decide neighbors. Taking the segment of component interaction trace shown in Fig. 3 (a), $\mathrm{C}_{3} \operatorname{rr}\left(\mathrm{I}_{\mathrm{r} 3}: \mathrm{I}_{\mathrm{p} 1}\right) \mathrm{C}_{2}$, for instance, the left neighbor of component $\mathrm{C}_{2}$ is $\mathrm{C}_{4}$ but not $\mathrm{C}_{3}$. Generally,

Rule 5: DMPI or DPI of a component cause DMRI or DRI of its left neighbor.

Rule 6: AMRI and ARI of a component cause AMPI or API of its right neighbor.

\section{Perform dynamic change impact analysis}

\subsection{Slices for component interaction trace}

Definition 8: Sub-component and super-component. Let $\mathrm{C}_{1}$ and $\mathrm{C}_{2}$ be two components. We call $\mathrm{C}_{1}$ is the subcomponent of $\mathrm{C}_{2}$ and $\mathrm{C}_{2}$ is the super-component of $\mathrm{C}_{1}$ if and only if $\forall \mathrm{I} \in \operatorname{Interface}\left(\mathrm{C}_{1}\right), \exists \mathrm{I}^{\prime} \in \operatorname{Interface}\left(\mathrm{C}_{2}\right)$, so that I' $\geq$ I.

From definition 8 , it is concluded that the subcomponent of component $\mathrm{C}$ would be obtained by deleting some interfaces or interface methods from it. The super-component of component $\mathrm{C}$ would be obtained by adding some interfaces or interface methods to it.

Definition 9: Interface Slicing Criterion. A interface slicing criterion for component interaction trace is a tuple with 3 elements $(\mathrm{C}, \mathrm{I}, \mathrm{X})$ in which $\mathrm{C}$ is a component, $\mathrm{I} \in$ Interface $(\mathrm{C})$, and $\mathrm{X} \in\{+,-\}$ indicating adding if $\mathrm{X}=$ "”+", or deleting if $\mathrm{X}=$ "“-”.

Definition 10: Method Slicing Criterion. A method slicing criterion for component interaction trace is a tuple with 4 elements $(\mathrm{C}, \mathrm{I}, \mathrm{M}, \mathrm{X})$ in which $\mathrm{C}$ is a component, $\mathrm{I} \in \operatorname{Interface}(\mathrm{C}), \mathrm{M} \in \operatorname{Method}(\mathrm{I})$, and $\mathrm{X} \in\{+,-\}$ indicating adding if $\mathrm{X}=$ "”+", or deleting if $\mathrm{X}=$ "“-".

Definition 11: Interface Slice. (1) A backward interface slice for component interaction trace $\mathrm{T}, \mathrm{S}_{\mathrm{ib}}$, on a given slicing criterion $(\mathrm{C}, \mathrm{I}, \mathrm{X})$ consists of all subcomponents and super-components of $\mathrm{C}$ in $\mathrm{T}$ that might directly or indirectly cause the addition or deletion of interface I; (2) A forward interface slice for component interaction trace $\mathrm{T}, \mathrm{S}_{\mathrm{ift}}$, on a given slicing criterion $(\mathrm{C}, \mathrm{I}, \mathrm{X})$ consists of all sub-components and super-components of $\mathrm{C}$ in $\mathrm{T}$ that might be directly or indirectly affected by adding or deleting interface I; (3) A unified interface slice for component interaction trace $\mathrm{T}, \mathrm{S}_{\text {iut }}$, on a given slicing criterion $(C, I, X)$ equals the combination of $S_{i b t}$ and $S_{\text {ift }}$, i.e. $\mathrm{S}_{\text {iut }}=\mathrm{S}_{\mathrm{ibt}} \cup \mathrm{S}_{\mathrm{ift}}$.
Definition 12: Method Slice. (1) A backward method slice for component interaction trace $\mathrm{T}, \mathrm{S}_{\mathrm{mbt}}$, on a given slicing criterion $(\mathrm{C}, \mathrm{I}, \mathrm{M}, \mathrm{X})$ consists of all sub-components and super-components of $\mathrm{C}$ in $\mathrm{T}$ that might directly or indirectly cause the addition or deletion of method $\mathrm{M}$ of interface I; (2) A forward method slice for component interaction trace $\mathrm{T}, \mathrm{S}_{\mathrm{mft}}$, on a given slicing criterion (C,I,M,X) consists of all sub-components and supercomponents in $\mathrm{T}$ that might be directly or indirectly affected by adding or deleting method $\mathrm{M}$ of interface I; (3) A unified method slice for component interaction trace $\mathrm{T}$, $\mathrm{S}_{\mathrm{mut}}$, on a given slicing criterion $(\mathrm{C}, \mathrm{I}, \mathrm{M}, \mathrm{X})$ equals the combination of $S_{m b t}$ and $S_{m f t}$, i.e. $S_{m u t}=S_{m b t} \cup S_{m f t}$.

With CIT, intra-component transition impact rule, inter-component transition impact rule, interface slicing and method slicing, we can determine all components, interfaces and methods which would cause given changes or are impacted by a given change. In section 4.2 , we illustrate the concrete analysis method with two examples.

\subsection{Determine impacted architectural elements}

To CIT $\mathrm{C}_{4}\left(\mathrm{I}_{\mathrm{r} 3}: \mathrm{I}_{\mathrm{p} 2}\right) \mathrm{C}_{1}\left(\mathrm{I}_{\mathrm{r} 1}: \mathrm{I}_{\mathrm{p} 3}\right) \mathrm{C}_{3} \operatorname{rr}\left(\mathrm{I}_{\mathrm{r} 3}: \mathrm{I}_{\mathrm{p} 1}\right) \mathrm{C}_{2}\left(\mathrm{I}_{\mathrm{r} 2}: \mathrm{I}_{\mathrm{p} 4}\right) \mathrm{C}_{3} \mathrm{rr}$, obtained in section 2.3, and a concrete change DPI(Ip2) of component $\mathrm{C} 1$, we could get a interface slicing criterion $\delta=\left(\mathrm{C}_{1}, \mathrm{I}_{\mathrm{p} 2},-\right)$. We perform interface backward slicing on slicing criterion $\delta$ to create a set consisting of the sub-components of those components in set $\left\{\mathrm{C}_{1}, \mathrm{C}_{2}, \mathrm{C}_{3}, \mathrm{C}_{4}\right\}$. Equally, we try to determine possible components and their interfaces whose changes would cause the deleting of $\mathrm{I}_{\mathrm{p} 2}$.

According to rule 3 and supposing there is dependency between $I_{r 1}$ and $I_{p 2}$ by referring to the mapping table among requirement methods and provider methods of component $\mathrm{C}_{1}$, it is concluded that $\operatorname{DRI}\left(\mathrm{I}_{\mathrm{r} 1}\right)$ of the same component $\mathrm{C}_{1}$ would cause the deleting of $\mathrm{I}_{\mathrm{p} 2}$. Then what change would cause the DRI $\left(\mathrm{I}_{\mathrm{r} 1}\right)$ ? According to Rule 5, change of $\operatorname{DPI}\left(\mathrm{I}_{\mathrm{p} 3}\right)$ on $\mathrm{C}_{1}$ 's right neighbor, $\mathrm{C}_{3}$ would cause $\operatorname{DRI}\left(\mathrm{I}_{\mathrm{r} 1}\right)$. Because component $\mathrm{C}_{3}$ doesn't have right neighbor, the impact transition stops. So we get all components, interfaces and methods that could cause the given change $\mathrm{DPI}\left(\mathrm{I}_{\mathrm{p} 2}\right)$ on component $\mathrm{C}_{1}$. Remaining these component and interfaces related to the change and removing those interfaces unrelated to that change, the trace's backward slicing on slicing criterion $\delta$ is $\mathrm{C}_{1}\left\{\mathrm{I}_{\mathrm{p} 2}, \mathrm{I}_{\mathrm{r} 1}\right\} \mathrm{C}_{3}\left\{\mathrm{I}_{\mathrm{p} 3}\right\}$. In fact, the stop of impact transition not only would be caused by return marks, but also would be caused by lack of dependency relationship in mapping table of provider interfaces and requirement interfaces.

\section{SOCIAT-software composition oriented change impact analysis tool}

The architecture of SOCIAT, which is shown in Fig. 5, includes 3 sub-systems: CIT generator, slicing performer 
and regression test suite scheduler. SOCIAT mainly has following three functionalities: (1) automatically generating component interaction traces according to a given change; (2) producing component set with indication of interfaces and methods that impact or be impacted by a given change; (3) scheduling regression test suite.

There are plenty of UML modeling tools that could be used to model static structure model of components composition and sequence diagram, such as Rational ROSE. Working principles of CIT generator and slicing performer have been illustrated in above sections. Regression test suite scheduler is used to verification that unchanged parts of system behavior same as before and changed parts of system behavior work as specification. The working principle of this sub-system is omitted in this paper because of the limitation of space.

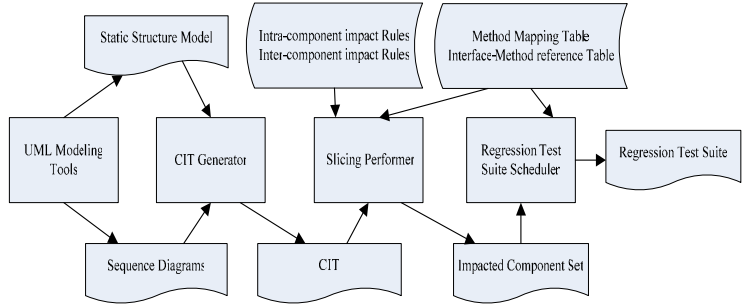

Fig.5. Architecture of SOCIAT

\section{Related works}

Previous research in change impact analysis of software system has been carried out with various approaches such as transitive closure on call graphs, static slicing, dynamic analysis and integration of static analysis and dynamic analysis. Although these classical impact analyses previous to ours provide some inspiration to our research, they only focus on code-level.

Jianjun Zhao [6] presented an approach to assess the effect of changes of software architecture by analyzing its Wright specification and to compute slices using flow graph-based technique.

L. C. Briand et al. [7] proposed a methodology supported by a prototype tool (iACMTool) to tackle the impact analysis and change management of analysis/design documents in the context of UML-based development. Opposite to our approach, their research focuses on consistency check among documents which is a kind of traceability analysis [1]. However, our approach belongs to the category of dependency analysis [1].

Another work that is similar to ours is that presented by Stafford et al. [8], who introduced a software architecture dependence analysis technique called chaining to support software architecture development such as debugging and testing. However, in contrast to our intra-component and inter-component impact rules based slice, they used a table-based approach to identify the chain sets.

\section{Conclusion}

An important problem demanding prompt solution with today's software development is to deal with the changes of software products. In this paper, a dynamic change impact analysis at software architecture level is proposed to support software architecture evolution.

The main contributes of this paper are listed as follows:

(1) Composition based software architecture is presented.

(2) Changes and impacts on composition based software architecture are explored.

(3) Interface slicing and method slicing based dynamic change impact analysis procedure is proposed.

(4) The architecture of SOCIAT, software composition oriented change impact analysis tool, is designed to support our approach.

\section{References}

[1] Bohner SA, Arnold RS, Software Change Impact Analysis, IEEE Computer Society Press: Los Alamitos CA, 1996.

[2] Shaw Mary, Garlan David. Software Architecture: Perspective on an Emerging Discipline. Prentice-Hall: Englewood Cliffs NJ, 1996.

[3] David Hemer, A formal approach to component adaptation and composition, Proceedings of the Twenty-eighth Australasian conference on Computer Science, Newcastle, Australia, Pages: 259 - 266, 2005

[4] Steffen Göbel, Encapsulation of structural adaptation by composite components, Proceedings of the 1st ACM SIGSOFT workshop on Self-managed systems, Newport Beach, California, Pages: $64-68,2004$.

[5] Luca de Alfaro and Thomas A. Henzinger, "Interface Theories for Component-Based Design", Proceedings of the First International Workshop on Embedded Software (EMSOFT), Lecture Notes in Computer Science 2211, SpringerVerlag, 2001, pp.148-165.

[6] Jianjun Zhao, Hongji Yang, Liming Xiang and Baowen Xu, Change impact analysis to support architectural evolution, Journal of Software Maintenance and Evolution: Research and Practice, Vol. 14, pp. 317-333, 2002.

[7] L. C. Briand, Y. Labiche, L. O'Sullivan, "Impact analysis and change management of UML models", Proceedings of the International Conference on Software Maintenance (ICSM'03), Amsterdam, Netherlands, pp.256-265, Sep. 2003.

[8] J.A. Stafford, A.L. Wolf and M. Caporuscio. "The Application of Dependence Analysis to Software Architecture Descriptions", Lecture Notes in Computer Science, Vol. 2804 Bernardo, Marco; Inverardi, Paola (Eds.) 2003, pp. 52-62. 